\title{
Rabaska
}

Revue d'ethnologie de l'Amérique française

\section{La recherche ethnologique au Centre de recherche bretonne et celtique, à Brest}

\section{Jean-François Simon et Fañch Postic}

Volume 13, 2015

Présence de Marius Barbeau : l'invention du terrain en Amérique

française. Autour d'un legs centenaire (1914-2014)

URI : https://id.erudit.org/iderudit/1033770ar

DOI : https://doi.org/10.7202/1033770ar

Aller au sommaire du numéro

Éditeur(s)

Société québécoise d'ethnologie

ISSN

1703-7433 (imprimé)

1916-7350 (numérique)

Découvrir la revue

Citer cet article

Simon, J.-F. \& Postic, F. (2015). La recherche ethnologique au Centre de recherche bretonne et celtique, à Brest. Rabaska, 13, 299-306.

https://doi.org/10.7202/1033770ar d'utilisation que vous pouvez consulter en ligne. 


\title{
La recherche ethnologique au Centre de recherche bretonne et celtique, à Brest
}

\author{
Jean-François Simon et Fañch Postic \\ Université de Bretagne occidentale, Brest
}

En 2014, le Centre de recherche bretonne et celtique (CRBC) est à la fois une structure de recherche, Équipe d'accueil (ÉA 4451) relevant du ministère de la Recherche et de l'enseignement supérieur, et une unité de documentation ou Unité mixte de service (UMs 3554) dépendant du même ministère mais aussi du Centre national de la recherche scientifique (CNRs). L'Équipe d'accueil regroupe des enseignants-chercheurs de l'Université de Bretagne occidentale (UBO) à Brest, son site historique - aujourd'hui à la faculté Victor-Segalen ${ }^{1}$ - et, depuis 2008, des collègues de l'Université de haute Bretagne (UHB) à Rennes. Elle est pluridisciplinaire et comprend des historiens, des linguistes celtisants et anglicistes, des littéraires, des sociologues et donc des ethnologues. Tous ont en commun de porter un intérêt scientifique à la Bretagne et aux pays celtiques, à ses sociétés du présent et du passé.

En ce sens, il s'y exprime une fidélité au projet d'origine : dès sa création en 1969, en effet, la recherche y est conduite « selon une perspective essentielle de pluridisciplinarité, dans des domaines tels que l'ethnographie, la géographie, l'histoire et l'archéologie, les langues et littératures bretonnes et celtiques. ${ }^{2} \gg$

Le fondateur de l'équipe, Yves Le Gallo, titulaire de la chaire de Civilisation de la Bretagne, précise ce qui est alors entendu par « ethnographie » : « Monsieur Jean-Michel Guilcher, explique-t-il, anime cette recherche. Monsieur Guillaume Floch, ethnographe, [...] étudie sur le vif, c'est-à-dire en naviguant, les techniques de pêche traditionnelle (en l'occurrence celle du

1. Centre de recherche bretonne et celtique, Université de Bretagne Occidentale, 20, rue Duquesne - CS 93837 - 29238 Brest cedex 3 ; T+ 33 (0)2 98016331 ; www.univ-brest.fr/crbc ; twitter : (a)crbcbrest.

2. Archives du CRBC. « Monsieur Le Gallo élu Directeur du Centre de Recherches bretonnes et Celtiques créé à l’U.E.R. des Lettres », communiqué destiné à la presse [1969]. 


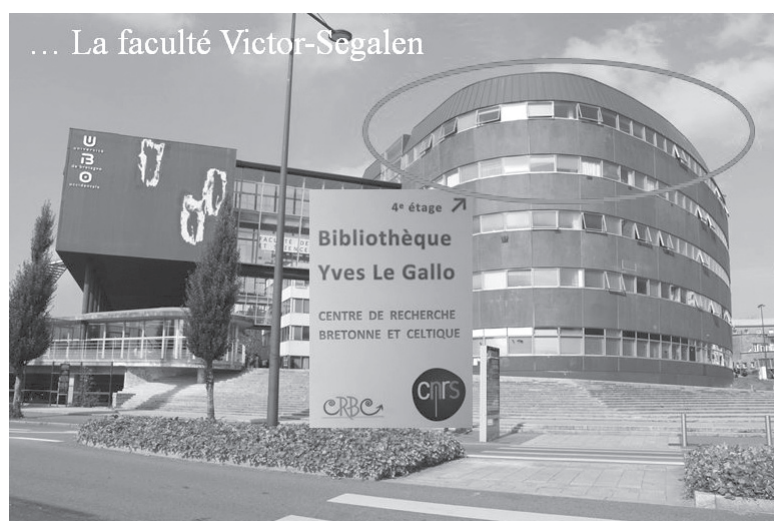

Centre de recherche bretonne et celtique.

Faculté Victor-Segalen

Université de Bretagne occidentale, Brest

maquereau de dérive). ${ }^{3} \gg$ On retrouve là, sous la terminologie hésitante d'un non-initié, l'ambition qui guide Jean-Michel Guilcher : il s'agit de collecter de toute urgence ce qui relève encore de la " civilisation traditionnelle » en Bretagne, ceci en s'en enquérant auprès des derniers dépositaires d'une certaine manière d'être au monde, qui n'a plus cours depuis 1914 mais qui survit dans les mémoires de ceux ayant connu cette époque.

Dans l'esprit de Jean-Michel Guilcher, la recherche envisagée (et engagée) vise surtout à collecter, dans la société bretonne du $\mathrm{Xx}^{\mathrm{e}}$ siècle finissant, ce qu'il reste d' " échantillons » de la « tradition orale à son terme »-c'étaient les mots dont il usait ${ }^{4}$.

Il se trouve que l'idée d'enquêtes à mener dans l'urgence préoccupe également les linguistes du CRBC, confrontés au recul irrémédiable de la langue bretonne ; le projet en vient même à " contaminer » les historiens qui déclarent eux aussi vouloir s'empresser de mener l'enquête orale en vue de procéder à « la récupération d'une "source historique" [qui] sauve de la disparition un matériel historique périssable et irremplaçable. ${ }^{5}$ » Les uns et les autres semblent alors se retrouver dans l'emploi récurrent qui est fait,

3. Archives du CrBC, Yves Le Gallo, «Projet de recherche présenté par le Centre de recherche bretonne et celtique de la Faculté des Lettres et Sciences sociales de Brest », s.d., [vers 1970].

4. Pour plus d'informations concernant l'implication de Jean-Michel Guilcher dans la création et l'organisation du CRBC entre 1969 et 1979, on se référera utilement à l'article des mêmes, « Du folklore à l'ethnologie : “"Traditions populaires” et projet universitaire en Bretagne. La contribution fondamentale de Jean-Michel Guilcher », dans le présent ouvrage. Voir aussi Jean-François Simon, « Les Enquêtes de Plozévet. Quelle place dans les recherches bretonnes en ethnologie du proche ? », En France rurale. Les enquêtes interdisciplinaires depuis les années 60 (Bernard Paillard, Jean-François Simon, Laurent Le Gall, dir.), Rennes, Presses universitaires de Rennes, 2010 : p. 189-206.

5. Archives du CRBC, « Section d'histoire. Projet de recherche. Évolution de la société bretonne de la fin du XIX ${ }^{e}$ au milieu du $\mathrm{Xx}^{\mathrm{e}}$ siècle» [vers 1970]. 
pour définir la démarche suivie, des termes « ethno-histoire » ou encore « ethnographie historique».

L'ambition est de procéder à terme à l'analyse de ce qui se décline à la manière « traditionnelle » dans la société bretonne en particulier et française en général : la « danse traditionnelle », le « chant traditionnel », « les fêtes traditionnelles », «l'architecture traditionnelle », etc. avec, pour visée finale, la compréhension du fonctionnement de la tradition, à travers ses contenus et les modalités de sa transmission.

Jean-Michel Guilcher est alors le seul à traiter de la « danse traditionnelle » : on ne se hasarde pas inconsidérément sur un terrain minutieusement balisé par le maître... Le rappel de deux dates suffit à traduire le plein investissement du chercheur dans un domaine tel que celui-ci : en 1963, paraît La Tradition populaire de danse en Basse-Bretagne ${ }^{6}$, en 2009, Danse traditionnelle et anciens milieux ruraux français. Tradition, histoire, société7.

$$
* * *
$$

Si l'on s'en tient à la chronologie, les ethnologues du CRBC furent d'abord des membres du Centre national de la recherche scientifique (CNRS) : JeanMichel Guilcher, Donatien Laurent, Fañch Postic. Se sont ensuite agrégés des universitaires, maître de conférences ou professeurs, dont le recrutement est justifié par l'institution d'un enseignement d'ethnologie à Brest, à partir de 1990 : ce sont successivement Patrick Le Guirriec, Jean-François Simon, Sergio Dalla Bernardina, Frédérique Ducharme, Marie-Armelle Barbier-Le Déroff, Camille Mazé, et enfin, au printemps dernier, la toute dernière recrue, Géraldine Le Roux. Nous sommes actuellement quatre ethnologues au CRBC.

Les recrutements successifs d'ethnologues à l'Université de Bretagne occidentale ont évidemment introduit de nouvelles problématiques de recherche au CRBC...

Il n'en reste pas moins que la leçon de Jean-Michel Guilcher continue de stimuler la réflexion. Si, dans les années 1970, la «tradition » qui lui était chère, n'existait déjà plus qu'à l'état d' « échantillons », il n'est pas difficile d'imaginer ce qu'il en est aujourd'hui... Pourtant, dans notre monde contemporain, nombreux sont ceux qui revendiquent encore une participation à une quelconque activité... « traditionnelle », à telle enseigne que, de qualificatif, le mot est devenu substantif : la forme nominale s'impose, le «traditionnel » a remplacé la « tradition ». À cet égard, nous rappelle opportunément JeanMichel Guilcher, «Une fois de plus l'affirmation pure et simple dispense d'y

6. Jean-Michel Guilcher, La Tradition populaire de danse en Basse-Bretagne, Paris-La Haye, Mouton, 1963.

7. Jean-Michel Guilcher, Danse traditionnelle et anciens milieux ruraux français. Tradition, histoire, société, Paris, L'Harmattan, 2009. 


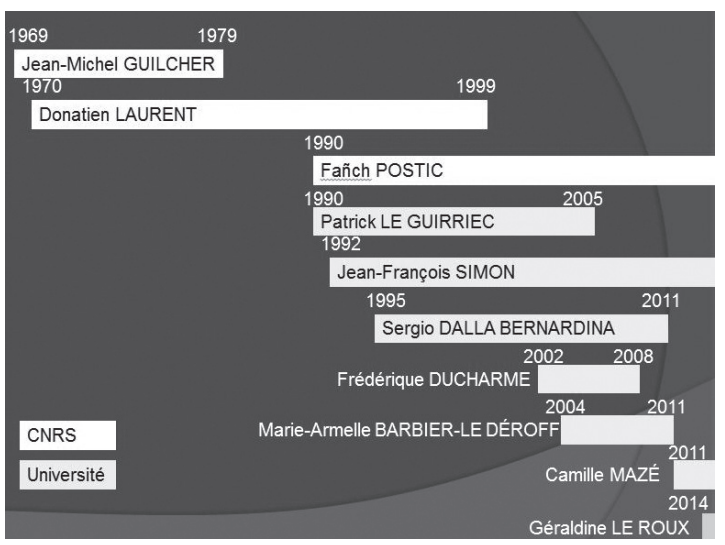

L'ethnologie au CRBC (1990-2014).

aller voir. Une vue simpliste en a remplacé une autre. ${ }^{8}$ » L'ambition demeure donc aujourd'hui « d'aller voir » ce qui se cache derrière la référence contemporaine au « traditionnel », à travers notamment la revendication identitaire et la valorisation patrimoniale?

Donatien Laurent, qui a suivi à Brest Jean-Michel Guilcher, son directeur de recherche, prend pour domaine de recherche la littérature orale et plus particulièrement la poésie chantée. La thèse qu'il soutient en 1974 sur le Barzaz-Breiz de Théodore Hersart de La Villemarqué, se fondant sur l'étude du premier carnet manuscrit de collecte et le confrontant à la bibliographie disponible et aux résultats de ses propres enquêtes sur le terrain, lui valent une notoriété internationale. La littérature orale devient l'un des axes importants de la recherche ethnologique au CRBC, laboratoire dont Donatien Laurent sera par ailleurs le directeur de 1987 à 1999. On peut mentionner les travaux de Michel Oiry (collectes de Le Diberder), Fañch Postic (collectes de François Cadic), Alain Tanguy (collectes d'Anatole Le Braz), Daniel Giraudon (chansons sur feuilles volantes et genres courts), etc.

La notoriété de Donatien Laurent a certainement contribué à l'ouverture d'un enseignement d'ethnologie à l'Uво à la rentrée universitaire de 1990. Elle l'a également conduit à établir des liens scientifiques avec différentes institutions qui, tant en Europe qu'en Amérique du Nord, partagent les mêmes centres d'intérêt : ainsi sa rencontre avec Jean-Pierre Pichette et d'autres

8. Jean-Michel Guilcher, Danse traditionnelles et anciens milieux..., op.cit., p. 240, note 45.

9. Ce point de vue a été développé par Jean-François Simon lors de la table ronde « Jean-Michel Guilcher, enseignant-chercheur à Brest (1969-1979) au carrefour de l'UBO, du CRBC, de la Société d'ethnologie bretonne et de l'ÉHÉss », organisée le 15 novembre 2014 à la Faculté Victor-Segalen à Brest, à l'occasion du Jubilé scientifique de Jean-Michel Guilcher. Le propos sera partiellement repris dans Ethnologie française (à paraître : vol. xLv, nº 3, 2015, p. 541-578 : p. 555). 
collègues franco-canadiens en 1991 à Sudbury (Ontario) lors du colloque consacré à l'œuvre du père Germain Lemieux, va marquer le début de toute une série de rencontres qui ont jalonné les vingt-cinq dernières années, à l'exemple du double colloque consacré à « L'apport des prêtres et de religieux au patrimoine des minorités » qui s'est tenu du 19 au 21 octobre 2011 à l'Université Sainte-Anne à Pointe de l'Église (Nouvelle-Écosse) et les 17 et 18 novembre à $1^{\prime} \mathrm{UBO}^{10}$. Le présent colloque est donc un nouveau maillon qui vient s'ajouter à cette chaîne déjà longue de " parcours comparés de la Bretagne et du Canada français ».

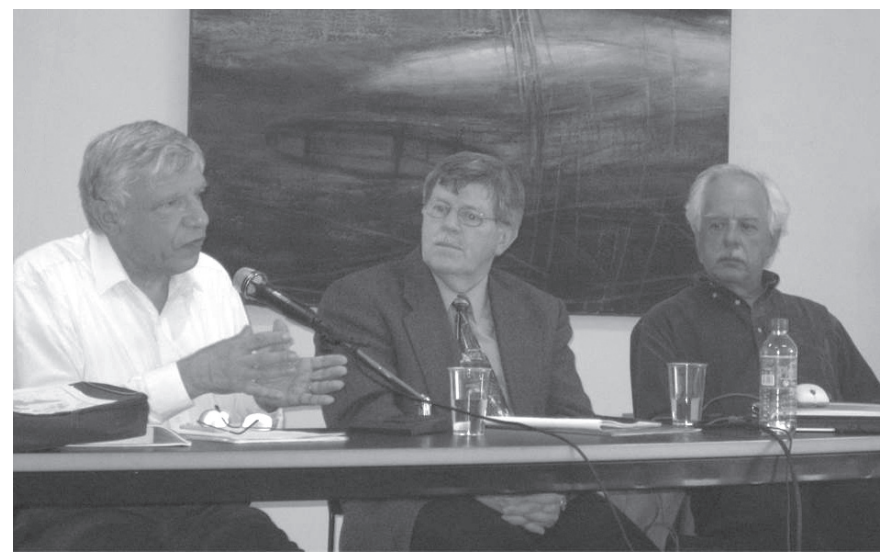

Donatien Laurent, Jean-Pierre Pichette et Jean Simard.

Premières rencontres britto-franco-canadiennes organisées par le CRBC :

Traditions, revitalisations, créations (Brest, 5-6 juin 2006)

C'est encore La Villemarqué, la littérature orale et Donatien Laurent qui sont à l'origine de la création, au sein du domaine départemental de Kernault, situé à Mellac, dans le sud du Finistère, d'une antenne du Centre de recherche bretonne et celtique. En 1989, la municipalité de Mellac, relayée par le département du Finistère, mène une réflexion quant à l'acquisition d'un ancien manoir mis en vente sur la commune. Se pose très vite la question de la vocation du futur site départemental : or, en 1989, le $150^{\mathrm{e}}$ anniversaire de la première édition du Barzaz-Breiz donne lieu à divers événements dont l'édition de la thèse de Donatien Laurent. Dès janvier 1990, l'idée se fait jour de la création d'une antenne du CRBC consacrée à l'étude de la littérature orale. Des contacts sont pris avec la famille La Villemarqué qui accepte de mettre le fonds d'archives familiales à la disposition du futur centre. Le Centre

10. Les actes en ont été réunis sous la direction de Jean-Pierre Pichette dans Port-Acadie, Revue interdisciplinaire en études acadiennes, n 24-25-26, automne 2013, printemps-automne 2014. 
de recherche et de documentation sur la littérature orale, ouvert au premier juillet 1990, est officiellement créé en 1991 selon une convention signée par le département du Finistère, l'Université de Bretagne occidentale et le Centre national de la recherche scientifique. La responsabilité en est confiée à Fañch Postic, ingénieur d'étude au CNRS.

Le travail documentaire du Centre porte, bien entendu, sur les archives La Villemarqué, mais aussi de manière plus générale, sur les collectes et les collecteurs en Bretagne depuis la fin du XVIII ${ }^{\mathrm{e}}$ siècle, replacés dans le cadre d'un mouvement européen d'intérêt pour la littérature orale. Cela donnera lieu, à Kernault, à des rencontres scientifiques internationales, à l'exemple de « La Bretagne et la découverte de la littérature orale en Europe ${ }^{11}$ », colloque organisé en 1995 à l'occasion du centenaire de la mort de l'auteur du BarzazBreiz, célébration qui est également l'occasion d'une importante exposition, les « Passeurs de mémoire » ${ }^{12}$. Par la suite, l'oralité demeurera le principal axe des animations et expositions proposées au domaine de Kernault : depuis plusieurs années, on parle d'ailleurs de parcours sonores, dont le dernier en date, « De bouche à oreille. Des histoires à dévorer », présenté d'avril à novembre 2014, s'est appuyé sur une présentation des répertoires breton et français comparés avec ceux des francophonies d'Amérique du Nord, puisant largement dans les collectes réalisées par Jean-Pierre Pichette. On y trouvait également des documents empruntés à d'autres collecteurs, dont Marius Barbeau, sujet du présent colloque. Repris par le musée François-Pilote de La Pocatière, ce parcours sonore sera présenté au public québécois à partir de juin 2015.

L'objectif premier du centre de Kernault, qui était de mieux cerner le rôle important de la Villemarqué et des collecteurs bretons dans l'avènement en France, et au-delà en Europe, d'un nouveau champ de recherche, la littérature orale, trouve son prolongement dans toute une série de rencontres scientifiques organisées par le CRBC et différents partenaires institutionnels ou associatifs : Émile Souvestre à Morlaix (Finistère) en 2006, Jean-Marie de Penguern à Lannion (Côtes-d'Armor) en 2007, Jacques Cambry à Quimperlé (Finistère) en 2008, Paul Sébillot à Fougères (Ille-et-Vilaine) en 2009, François Cadic à Pontivy (Morbihan) en 2010, René-Yves Creston à Batz-sur-Mer (LoireAtlantique) et, à nouveau, Théodore Hersart de la Villemarqué à Quimperlé en 2015. Ce sont autant d'occasions de scruter le passage du folklore à l'ethnologie en Bretagne.

11. La Bretagne et la littérature orale en Europe, actes du colloque international de mai 1995, Brest Circto/CrвB, 1999.

12. Cf. Donatien Laurent, Fañch Postic, Pierre Prat, Les Passeurs de mémoire, Manoir de Kernault, 1996. 
En 1976, la création d'un Centre d'ethnologie de la France à Brest, antenne de l'ÉHÉss, s'était accompagnée de la constitution d'un fonds documentaire. Faute de voir se créer un poste pour en assurer le fonctionnement, ce fonds a été intégré dans les collections de la bibliothèque du CRBC où il a continué de s'enrichir même après la fermeture du Centre d'ethnologie de la France. Reconnue comme unité mixte de service (UMS) depuis le $1^{\text {er }}$ septembre 2012 par le CNRS, la bibliothèque, qui porte désormais le nom du fondateur du CRBC, Yves Le Gallo, dispose d'un fonds conséquent relatif à l'ethnologie de la Bretagne et de la France. Elle s'est également enrichie de dépôts importants d'archives : les carnets d'Anatole Le Braz (plus d'une centaine), le fonds de l'abbé François Falc'hun (qui fut l'un des participants à l'enquête sur le folklore musical en Bretagne en 1939), le fonds Robert Gessain (enquêtes de Plozévet), le fonds sonore de Donatien Laurent (les bandes ont été numérisées et sont en cours de documentation).

$$
* * *
$$

Pour illustrer les travaux à caractère ethnologique menés au CRBC, nous avons choisi d'évoquer les thèses qui y ont été soutenues, d'abord dans le cadre d'un partenariat entre l'Université de Bretagne occidentale et l'École des hautes études en sciences sociales (ÉHÉss), puis, depuis 1982, sous le couvert de l'École doctorale de Brest. Les thèmes de recherche, divers et variés, sont à l'image des thématiques et des méthodes caractérisant la polymorphie actuelle de la recherche ethnologique, en France comme ailleurs.

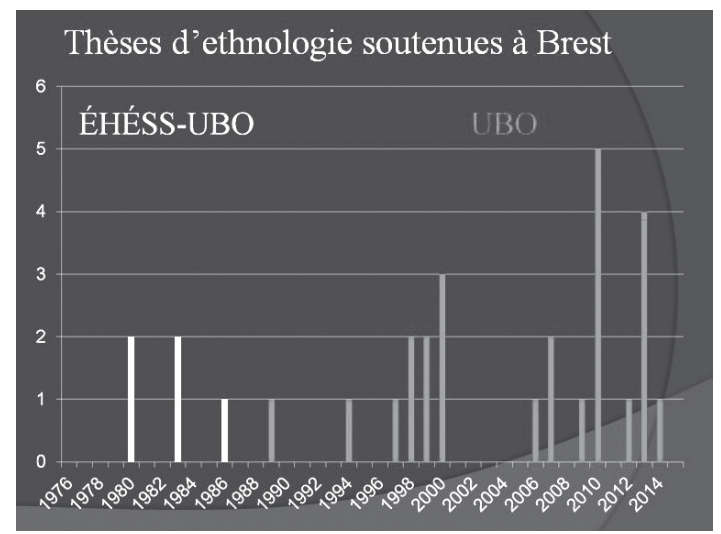

Thèses d'ethnologie soutenues à Brest (1980-2014).

Pour terminer cette rapide présentation de la recherche ethnologique menée au CRBC, nous voudrions évoquer rapidement quelques dates qui ont 
marqué l'histoire de l'enseignement de l'ethnologie à Brest, depuis la création du premier enseignement en 1982, jusqu'à ce jour où s'élabore l'offre de formation pour le prochain plan quinquennal :

1982 : Création d'une unité d'enseignement d'Ethnologie de la Bretagne dans le cadre d'une licence de Civilisation de la Bretagne.

1990 : Création d'une licence d'Ethnologie (généraliste), suivie l'année suivante de la création d'une maîtrise.

1992 : Ouverture au concours d'un premier poste d'enseignant-chercheur (maître de conférences).

2004 : Création d'un master 2 professionnalisant Concepteur de projets en tourisme culturel.

2012 : Création d'un Parcours d'ouverture pluridisciplinaire (PoP) qui permet de faire figurer des enseignements d'ethnologie en L1 et L2, dans le cadre des licences de sociologie et de philosophie.

Actuellement, à l'Université de Bretagne occidentale, l'ethnologie est enseignée à tous les niveaux : licence 1 (L1), licence 2 (L2), licence 3 (L3), master 1 (M1), master 2 (M2) recherche, master 2 professionnalisant, ces dernières formations étant adossées au CRBC et susceptibles de fournir des doctorants auxquels viennent tous les ans s'agréger des étudiants intéressés par les problématiques développées par le CRBC. En 2017 sera présentée à la validation du Ministère une nouvelle offre de formation qui, si elle est approuvée, sera effective pour les cinq années suivantes. 